\title{
Umanizzazione delle cure: innovazione e modello assistenziale
}

\author{
Francesco Burrai ${ }^{1}$, Luigi Apuzzo ${ }^{2}$, Valentina Micheluzzi ${ }^{3}$ \\ ${ }^{1}$ SC Formazione, Ricerca e Cambiamento Organizzativo, ATS Sardegna - ASSL di Sassari, Sassari - Italia \\ ${ }^{2}$ Infermiere Olistico, AOU di Sassari, Sassari - Italia \\ ${ }^{3}$ Ricercatore, Centro di Ricerca SIAN, Santa Maria Capua Vetere, Caserta - Italia
}

\begin{abstract}
Humanization of Care: Innovation and Health Care Model
The contemporary social context shows a profound need to bring healthcare back to a meeting between the technical-organizational needs of the system and the needs for global understanding of the patient. The passage from the "patient" vision to a "person" perspective is the radical request for paradigmatic and operational change to be implemented to respond to new needs, trends and socio-cultural changes. Recent literature shows that the Newtonian-Cartesian reduction of the human being to biomachine, which still resists the culture and unconscious of many health professionals, is no longer justified from a scientific point of view. The conceptual model, the framework of the most advanced sciences is the holistic one. This model lays the foundations for a better understanding of the functioning of the human being and consequently for a new epistemological and anthropological vision, that of the bio-psycho-socio-cultural network, where interaction with the environmental context influences the feedback of this system. It seems fundamental to start a structured path of synergy between the humanization of care and its gradual implementation, for the improvement of the quality of life of nephrological patients and for a new clinical-assistance background for the health workers who work daily in nephrology, dialysis and transplantation.
\end{abstract}

Keywords: Health care model, Holistic care, Humanization of care, Innovation

\section{Introduzione}

Nei Paesi sviluppati, negli ultimi anni, il management sanitario ha introdotto nel policy making l'umanizzazione delle cure (UC), come strategia fondamentale per rispondere in maniera innovativa e sinergica al contesto sociale ed epidemiologico e alle nuove richieste e ai bisogni di salute della popolazione, che richiedono un adeguamento delle risposte assistenziali sia sul piano clinico-assistenziale sia su quello organizzativo-gestionale.

Nell'era contemporanea, le persone possiedono un più alto livello culturale e, quando accedono al mondo sanitario, chiedono di non subire più quel processo disumanizzante in

Received: January 21, 2020

Accepted: March 8, 2020

Published online: April 26, 2020

Indirizzo per la corrispondenza:

Francesco Burrai

SC Formazione, Ricerca e Cambiamento Organizzativo

ATS Sardegna - ASSL di Sassari

Strada Statale 200

07100 Sassari - Italia

francesco.burrai@atssardegna.it cui la loro esperienza di sofferenza o di malattia venga ridotta alla sola identificazione tecnica di un segno, di un sintomo, di un dato biochimico o di un organo malato.

Questa visione dell'individuo da parte di molti sanitari è dovuta a una percezione dell'essere umano solo come una complessa bio-macchina, portatrice di un "guasto", che deve essere "riparato" solo con opportune somministrazioni di molecole o interventi chirurgici. Questa condizione spersonalizza l'individuo e lo svalorizza, perché, in tale approccio, non ha importanza "clinica" ciò che la persona percepisce, intuisce e "sente" della sua condizione esistenziale.

Questa realtà ha condotto e conduce tuttora a pregiudizi e conflitti tra la popolazione e il mondo dei sanitari, di cui I'incremento delle controversie giudiziarie, l'incremento progressivo delle polizze assicurative tra i sanitari e la medicina difensiva sono degli esempi.

La società di oggi, dunque, accetta sempre meno di subire un intervento sanitario in cui non è contemplata la considerazione della persona come essere umano globale e, in tale nuovo contesto culturale e sociale, appare strategico offrire alla popolazione un'assistenza sanitaria olistica, dunque non più caratterizzata solo da attività tecniche e farmacologiche, ma anche da interventi non farmacologici, di humanities, che possano rispondere ai nuovi bisogni dell'utenza di oggi. 
Il management dell'UC risponde a tale esigenza contemporanea, la cui implementazione richiede una metodologia sistemica, per processi, che permetta di pianificare, organizzare, dirigere, coordinare e monitorare le attività connesse all'umanizzazione delle cure.

In Italia, a livello dei policy maker sanitari, si sta assistendo a un'attività di politica sanitaria che cerca di porre sempre maggiore attenzione e dedicare sempre più risorse all'UC, introducendo nuove competenze di public management, orientate a una strategia di change management, che ha l'obiettivo di creare un'integrazione multiprofessionale e multidisciplinare, rispetto alle modalità di attivazione degli strumenti di umanizzazione delle cure.

L'UC richiede l'utilizzo: a) dell'informational continuity, ovvero l'uso delle informazioni relative alla storia clinica di un paziente per selezionare le opzioni più appropriate circa il suo percorso assistenziale; b) della management continuity, ovvero l'attenzione e la presa in carico dei bisogni sociosanitari spesso in costante cambiamento di un paziente; c) della relational continuity, ovvero la relazione personale iterativa tra un paziente e uno o più professionisti.

Nel management sanitario moderno, le UC interessano le configurazioni dell'assetto di governance a livello di Hub \& Spoke dell'assistenza ospedaliera, il livello territoriale e la home care. Gli aspetti manageriali, in questo settore, impattano sul disease management, integrando il know-how clinico con il know-how dell'UC, sugli outcome clinici, sulla customer satisfaction e sulla compliance del paziente, integrando il case management e il counseling con gli strumenti innovativi dalla telemedicina e del teleconsulto.

\section{Il paradigma concettuale olistico}

L'umanizzazione delle cure si basa sul paradigma olistico. Il termine olismo deriva dalla parola greca holos

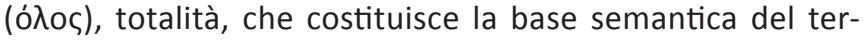
mine moderno "olismo". Nel paradigma olistico le proprietà di un sistema non possono essere derivate solo attraverso la conoscenza prodotta dalle informazioni delle singole componenti e, in base alla teoria dei sistemi, il tutto possiede un significato maggiore e diverso dalla semplice somma delle parti (1).

Applicata all'essere umano, la comprensione fenomenologica, epistemologica e funzionale è legata alla condizione di integrazione e intercorrelazione delle parti, senza l'uso di una metodologia riduzionistica di stampo meccanicistico, newtoniano-cartesiano. Nell'uomo, il network è formato da componenti biologiche, psichiche, spirituali, culturali e sociali connesse con l'ambiente, in un'interconnessione inscindibile tra le componenti, in cui flussi di informazioni hanno caratteristiche circolari e dinamiche con feedback tra le informazioni, dove ogni cambiamento in uno di questi livelli influenzerà simultaneamente e istantaneamente tutte le altre parti che compongono il sistema uomo-ambiente (2).
Le implicazioni filosofiche, epistemologiche e pragmatiche per l'assistenza sanitaria sono rilevanti. Storicamente e per natura intrinseca, il sanitario è olistico, ma le condizioni organizzative e culturali hanno modificato questa natura, a favore di un'assistenza legata non più alla visione dell'uomo in senso globale, ma a una visione riduzionistica, dove il focus è solamente il corpo malato, che ha bisogno di essere "riparato" in una sua parte specifica, e, di conseguenza, a favore di un'assistenza specializzata all'organo, non alla persona. Allora, l'essere umano diventa una macchina-corpo, che necessita solo di riparazioni, e la malattia e la disabilità sono considerate come entità oggettive, separabili, misurabili e modificabili, che non possono essere influenzate da pensieri e da emozioni.

Nel modello olistico, il dolore e la malattia hanno anche, nella componente psicosomatica, biologica, psicologica, sociale e spirituale, i loro fattori eziologici. L'intervento assistenziale olistico si basa sulla conoscenza e sulla consapevolezza che l'essere umano è un'interconnessione di corpo, mente, spirito ed energia, in cui le componenti sociale, culturale, relazionale e ambientale giocano ruoli importanti nel mantenimento della salute e del benessere individuale. Per tale ragione, l'azione assistenziale olistica è indirizzata verso l'individuazione delle risorse e del potenziale terapeutico e rigenerante del sistema mente-corpo.

II mondo dei sintomi e dei segni non è più interpretato in maniera riduttiva come solo dato clinico, che richiede un trattamento specifico secondo il modello cartesiano e newtoniano, ma come nuovo linguaggio, forma di comunicazione del tutto e del sistema uomo, con significati molto più ampi, che può condurre alle vere cause del problema, che non sono mai univoche e uniche.

La salute non è definita da un'assenza di malattia, dualità tipica del modello biomedico, ma da una condizione di stato, da una qualità di condizione intesa come integrazione ed equilibrio del sistema individuale interconnesso con l'ambiente.

Ogni persona diventa un sistema irripetibile, non riproducibile e diverso da ogni altro individuo, perché la complessità individuale e la sua continua trasformazione nello spaziotempo non permetteranno mai di identificare individui con le medesime caratteristiche, ed ogni soggetto ha percezioni peculiari del mondo interiore ed esteriore, percezioni che mutano nel loro divenire esistenziali, allontanando, così, ogni possibile tentativo di definizione che punti a dare una determinazione e una descrizione completa di una percezione umana.

Ogni persona possiede capacità, risorse ed energie che conducono al miglioramento della sua condizione, fino alla possibile guarigione, e tali capacità sono innate. L'azione dell'assistenza olistica ha l'obiettivo di aiutare la persona a identificarle, indirizzarle, potenziarle e, soprattutto, usarle.

\section{Management sanitario e umanizzazione delle cure}

L'UC è una vision manageriale legata al framework concettuale olistico, in cui i risultati delle attività sanitarie, non sono 
solo legati ai trattamenti biomedici e all'assistenza standard, ma anche all'utilizzo di trattamenti non farmacologici e a un'organizzazione fortemente personalizzata, in cui il cittadino è considerato in maniera globale, come un essere umano con $\mathrm{i}$ suoi bisogni, non solo fisici, ma anche psicologici e spirituali.

L'UC è complementare e sinergica con la tecnologia e cerca di mantenere costante nell'organizzazione il concetto di persona e non di paziente, di cittadino e non di corpo da trattare, di essere unico e non di codice a barre o posto letto.

L'UC accoglie le esigenze sia quotidiane che di self-care della persona, cercando di introdurre un sistema di accoglienza e un sistema sanitario, assistenziale "umano", di vicinanza e di comprensione, attraverso strategie, metodi e strumenti moderni e scientifici.

Questa strategia di management ha un'azione non solo verso l'esterno, ma anche verso l'interno, vale a dire verso le professionalità che compongono l'organizzazione, soprattutto verso i sanitari. Infatti, secondo il paradigma olistico dell'UC, non può esserci una sanità capace di soddisfare i bisogni dei cittadini e capace di aumentare il benessere e la qualità di vita e di migliorare lo stato di salute della persona, se le professionalità e le persone dell'organizzazione sanitaria non hanno, a loro volta, uno stato di benessere, una buona qualità di vita e una migliore condizione di salute. Questo è estremamente evidente nella sanità contemporanea, dove la spersonalizzazione dell'assistenza e la sequenzialità burocratico-amministrativa hanno reso molti settori sanitari e, dunque, gli stessi sanitari soggetti di procedure amministrative e burocratiche che hanno allontanato queste professionalità dai cittadini.

Questo allontanamento, associato all'aumento dei carichi di lavoro, al tipo dei nuovi carichi (burocratizzazione, controllo budget, mero lavoro di segretariato, ecc.), alla riduzione del personale e ai tagli alla sanità di vario tipo, ha drasticamente ridotto la qualità di vita dei sanitari. Conseguentemente, si assiste a un innalzamento di malessere, insoddisfazione, probabilità di errori e conflitti con i pazienti, con i familiari e dentro l'equipe, portando a un clima organizzativo certamente non umanizzante, accogliente e pronto a soddisfare i bisogni di salute psicofisica.

In questo contesto di spersonalizzazione e di disumanizzazione dell'assistenza a un processo di riduzione del paziente a una patologia, organo, numero, ID, posto letto, codice a barre e così via, si è innescata la medicina difensiva: l'esplosione delle denunce alle autorità giudiziarie e le segnalazioni all'URP e al Tribunale dei diritti del malato e, non ultimo, articoli sui giornali, reportage televisivi e post di qualunque tipo sul web stanno portando a una progressione delle polizze assicurative dei sanitari, da un lato e all'aumento delle richieste di accertamenti dall'altro.

L'umanizzazione delle cure è strettamente collegata alla "Qualità" e, concettualmente, al ciclo di Deming, dove, nell'applicazione dell'approccio per processi, il primo principio delle norme ISO 9000:2000 del Sistema di Gestione per la Qualità pone come pilastro fondamentale l'organizzazione mettendo in primo piano i propri pazienti, e di conseguenza, la necessità manageriale di comprendere le esigenze dei pazienti, non solo presenti ma anche future, perseguendo la soddisfazione dei loro requisiti, nonché di superare le aspettative del paziente.

II management sanitario dell'umanizzazione delle cure segue un sistema circolare di step collegati che garantisca il miglioramento continuo (Fig. 1).

Nello specifico, le azioni strategiche del management dell'umanizzazione delle cure sono individuate nella Tabella I.

L'UC può contribuire a raggiungere e a migliorare diversi outcome del sistema sanitario e dell'assistenza sanitaria (Tabella II).

Per il raggiungimento degli obiettivi strategici legati all'implementazione dell'UC è necessario strutturare 4 aree: 1) Area Formazione; 2) Area Ricerca; 3) Area Valutazione; 4) Area Clinico-Assistenziale. Ogni area presenta le specifiche azioni, come mostrato nella Tabella III.

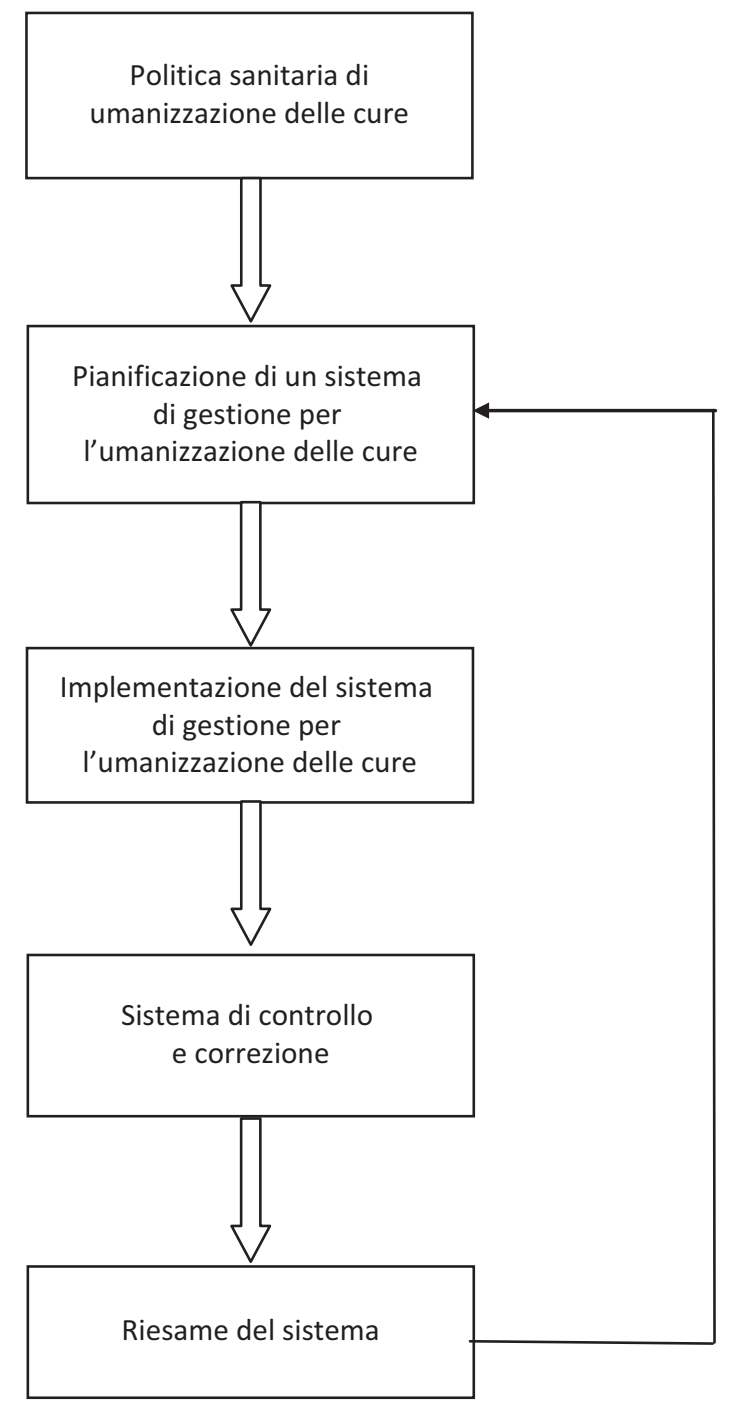

Fig. 1 - Sistema di management sanitario dell'umanizzazione delle cure. 
Tabella I - Azioni strategiche del management dell'umanizzazione delle cure

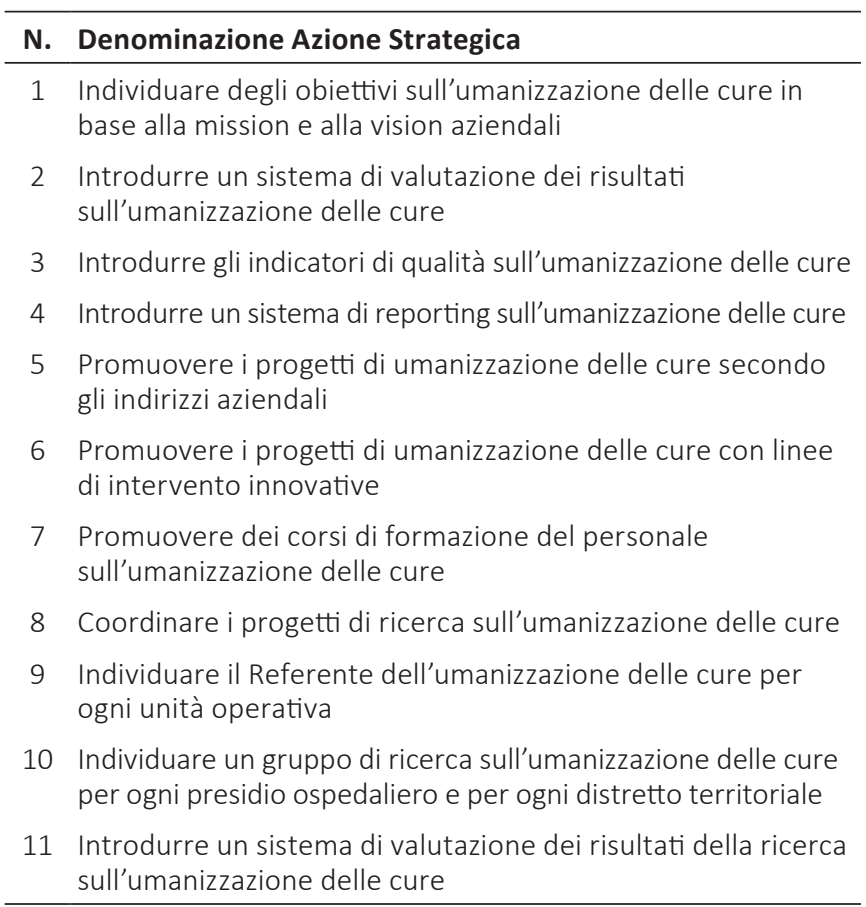

Tabella II - Impatto dell'Umanizzazione delle cure

\begin{tabular}{ll} 
N. & Tipologia di impatto dell'UC \\
\hline 1 & Garantire il conseguimento dei livelli essenziali di assistenza \\
in maniera omogenea & Migliorare il grado di soddisfazione degli utenti \\
3 & Ridurre la spesa farmaceutica \\
4 & Ridurre la spesa sociosanitaria \\
5 & Ridurre i contenziosi legali \\
6 & Ridurre gli errori \\
7 & Aumentare l'efficacia, l'efficienza, la sicurezza e l'appropriatezza \\
8 & Ridurre le complicanze \\
9 & Ridurre i tempi di degenza \\
10 & Migliorare le performance professionali \\
11 & Migliorare l'organizzazione \\
12 & Migliorare la relazione e la comunicazione sanitario-cittadino \\
13 & Migliorare il grado di soddisfazione degli operatori sanitari \\
14 & Ridurre il burn-out, il turn-over e il mobbing
\end{tabular}

Tabella III - UC e obiettivi strategici

\begin{tabular}{|c|c|c|c|}
\hline Area Formazione & Area Ricerca & Area clinico-assistenziale & Area Valutazione \\
\hline $\begin{array}{l}\text { Formazione ECM, di livello base, } \\
\text { sugli interventi di UC }\end{array}$ & $\begin{array}{l}\text { Progettazione e conduzione di } \\
\text { studi di ricerca di tipo qualitativo, } \\
\text { quantitativo e mixed method } \\
\text { nell'UC }\end{array}$ & $\begin{array}{l}\text { Implementazione } \\
\text { dell'umanizzazione delle cure } \\
\text { nella pratica clinico-assistenziale }\end{array}$ & $\begin{array}{l}\text { Strutturazione di strumenti di } \\
\text { misurazione e valutazione dell'UC }\end{array}$ \\
\hline $\begin{array}{l}\text { Formazione ECM, di livello avan- } \\
\text { zato, sugli interventi di UC }\end{array}$ & $\begin{array}{l}\text { Strutturazione di ricerche innova- } \\
\text { tive per i bandi di finanziamento } \\
\text { europei }\end{array}$ & $\begin{array}{l}\text { Revisione della pratica clinico- } \\
\text { assistenziale introducendo le tec- } \\
\text { niche olistiche presenti nell’UC }\end{array}$ & $\begin{array}{l}\text { Individuazione degli indicatori e } \\
\text { degli standard di efficacia delle } \\
\text { performance nell’UC }\end{array}$ \\
\hline $\begin{array}{l}\text { Formazione ECM sui sistemi di } \\
\text { implementazione delle UC }\end{array}$ & $\begin{array}{l}\text { Strutturazione di Linee Guida } \\
\text { sull'UC }\end{array}$ & $\begin{array}{l}\text { Revisione degli standard assisten- } \\
\text { ziali nella logica dell'UC }\end{array}$ & $\begin{array}{l}\text { Implementazione del sistema } \\
\text { degli indicatori }\end{array}$ \\
\hline $\begin{array}{l}\text { Formazione ECM sui sistemi } \\
\text { di misurazione e valutazione } \\
\text { delle UC }\end{array}$ & $\begin{array}{l}\text { Strutturazione di procedure e } \\
\text { protocolli di Evidence Based } \\
\text { Practice nell’UC }\end{array}$ & $\begin{array}{l}\text { Trasformazione delle UO in zone } \\
\text { di innovazione e sperimentazione } \\
\text { assistenziale nell'UC }\end{array}$ & $\begin{array}{l}\text { Misurazione e valutazione siste- } \\
\text { matica in base a un planning }\end{array}$ \\
\hline $\begin{array}{l}\text { Formazione Universitaria con } \\
\text { Master di primo livello in UC }\end{array}$ & $\begin{array}{l}\text { Divulgazione dei risultati delle } \\
\text { ricerche a livello nazionale e } \\
\text { internazionale }\end{array}$ & $\begin{array}{l}\text { Supervisione dell'assistenza } \\
\text { basata sull'Evidence Based } \\
\text { Practice nell'UC }\end{array}$ & $\begin{array}{l}\text { Reporting annuale dei livelli di } \\
\text { performance nell'UC }\end{array}$ \\
\hline
\end{tabular}

\section{Normativa nell'umanizzazione delle cure}

L'umanizzazione delle cure presenta un percorso legislativo in Italia, che parte dagli anni '90, attraverso il Decreto Legislativo 30 dicembre 1992, n. 502, art. 14, comma primo, in cui si espongono i concetti sottoposti a gestione manageriale di personalizzazione e umanizzazione delle cure (3). II Decreto Ministeriale 15 ottobre 1996 del Ministero della Sanità introduce anche un sistema di indicatori per la valutazione delle dimensioni qualitative del servizio, riguardanti la personalizzazione e l'umanizzazione dell'assistenza (4).

L'articolo 1, comma primo, del Decreto Ministeriale 15 ottobre 1996 prevede che il Servizio Sanitario Nazionale introduca un sistema di indicatori per la verifica della qualità dei servizi e delle prestazioni sanitarie, con riferimento alla personalizzazione e all'umanizzazione dell'assistenza. ॥ Decreto del Presidente del Consiglio dei Ministri 19 maggio 
1995 introduce la Carta dei servizi pubblici sanitari, la quale rappresenta un documento che descrive processi sottoposti a sviluppi e personalizzazione dell'assistenza (5). L'umanizzazione delle cure è legata ai concetti di trasparenza, con riferimenti alla Costituzione Repubblicana, all'articolo 3 nei principi di eguaglianza e all'articolo 97 nei principi di imparzialità e buon andamento della Pubblica Amministrazione (6) e, con la Legge 7 agosto 1990, n. 241, il principio di trasparenza dell'azione amministrativa è sancito in maniera esecutiva (7). Nel Patto per la Salute 2014-2016 in riferimento all'umanizzazione delle cure, c'è una previsione specifica al riguardo. Infatti, l'umanizzazione nell'organizzazione sanitaria garantisce il recupero della centralità del paziente, facendosi carico non solo degli aspetti fisici della malattia, ma anche di quelli psicologici, relazionali e sociali. II concetto di centralità del paziente nei servizi sanitari è stato, infatti, più volte affermato in questi anni nella normativa internazionale, nazionale e regionale e i diritti dei pazienti sono la meta prioritaria dei singoli Paesi e delle associazioni di pazienti (8). Nell'Atto di Indirizzo del 2017 del Ministero della Salute, si esplicita come gli obiettivi possono essere raggiunti anche potenziando gli aspetti di umanizzazione delle cure (9). Nell'Atto di Indirizzo, al punto 5.3 "Qualità dei servizi sanitari e umanizzazione delle cure", si esorta a proseguire nel percorso di umanizzazione delle cure, attraverso una compiuta analisi dei reali bisogni delle persone, dei contesti, delle relazioni tra persone e ambienti e dei processi cognitivi ed emotivi individuali e di gruppo, in modo da individuare i bisogni latenti e di contestualizzare le richieste esplicite. Si prevede, inoltre, la predisposizione di un programma annuale di umanizzazione delle cure, che comprenda la definizione di almeno un'attività progettuale in tema di formazione del personale e di un'attività progettuale in tema di cambiamento organizzativo, indirizzato prioritariamente alle seguenti aree assistenziali: Area critica, Pediatria, Comunicazione, Oncologia e Assistenza domiciliare.

\section{Formazione universitaria nelle UC}

L'implementazione sistematica e scientifica in base a Linee Guida e il modello dell'EBCP possono essere garantiti con una formazione specifica dei sanitari a un alto livello scientifico dall'introduzione di un Master di primo livello in Umanizzazione delle Cure. Il percorso formativo del Master dovrebbe essere caratterizzato da una componente teorica che trasmetta un background di tipo metodologico e sistemico, con un collegamento costante tra gli aspetti olistici e I'EBCP. Strategico è I'utilizzo dei framework più avanzati prodotti dalla ricerca contemporanea, come quello quantistico e PNEI. Fondamentale l'azione dell'acquisizione delle skill tecniche in riferimento agli interventi olistici evidence based e attraverso laboratori full immersion e il relativo tirocinio. In questo momento, in Italia manca tale tipo di formazione.

\section{Conclusioni}

Vision e mission di un'attività sanitaria contemporanea devono utilizzare gli strumenti di management legati all'umanizzazione delle cure, non solo per raggiungere gli obiettivi sociosanitari e assistenziali, ma anche per ridurre i contenziosi legali tra sanitari e pazienti, ridurre le segnalazioni negative che pervengono all'URP e ridurre gli articoli negativi che compaiono nei media e l'escalation delle richieste di accertamenti non necessari e degli importi assicurativi dei sanitari. Questi aspetti sono fondamentali in una società multimediatica, in cui l'impatto delle informazioni sull'opinione della società civile in riferimento alla qualità dei servizi sanitari deve essere governato in maniera strategica e l'umanizzazione delle cure è una di queste strategie.

In Italia, anche la normativa più recente, sia nazionale che regionale, introduce e sviluppa in maniera costante, nella produzione degli atti legislativi, riferimenti precisi sull'introduzione dell'UC, sulla gestione manageriale e sugli obiettivi da raggiungere.

L'UC viene introdotta nel contesto sanitario attraverso una serie di interventi evidence based che devono seguire una metodologia di pianificazione, organizzazione, direzione, coordinamento, monitoraggio e ricerca, nell'ottica della best practice.

La qualità di vita è un aspetto fondamentale per la vita di una persona, per un essere umano, soprattutto se è in una condizione di crisi, di malattia e di solitudine. Se un intervento non farmacologico in sinergia con i trattamenti convenzionali presenta miglioramenti in diversi outcome della qualità di vita di una persona, è un risultato eticamente, moralmente e scientificamente importante.

II management sanitario, come indicato dalla letteratura scientifica e dalla normativa, deve essere capace di unire gli aspetti "freddi" legati ai farmaci, alla chirurgia, alla tecnologia e alla burocrazia con quelli "caldi", legati all'uomo, inteso come essere vivente non identificabile solo con un numero, un costo, un ID, un marcatore biochimico e un referto diagnostico strumentale. Questo approccio è, oggi, perdente. La società di oggi, i cittadini che accedono al SSN hanno maggiori informazioni, hanno un livello culturale più elevato, fanno confronti e spesso mostrano preconcetti di "malasanità", proprio dovuti alla non considerazione come esseri umani, al fatto di essere trattati appunto solo come "pazienti", soggetti che devono attendere, il cui parere, la cui opinione, le cui capacità interiori e le cui risorse di self-care spesso non vengono minimamente considerati.

Umanizzare le cure è un ricercare iniziative capaci di migliorare il contatto umano tra operatore sanitario e utente, ma anche tra gli stessi professionisti, e questo può essere fatto se esistono una pianificazione e un'attuazione, in definitiva, se esistono una struttura e un servizio di umanizzazione delle cure che permettano anche di recuperare l'immagine pubblica della sanità presso la società. 
Umanizzare è un processo che interessa il concetto di dignità. La sofferenza e il dolore di una persona che ha problemi di salute possono essere trattati con la capacità, da parte dei sanitari, di "sentire" l'altro, di parlare e di capire quali sono le risorse della persona: dunque, la persona non è più un soggetto passivo di trattamenti sanitari, ma attivo, che ha bisogno di sentirsi integrato e le sue risorse possono essere inglobate per un migliore self-care, per l'empowerment.

La sfida contemporanea del management sanitario è la trasformazione, in tutti i processi sanitari, ospedalieri e territoriali, del nostro utente da soggetto passivo, da trattare e che subisce l'organizzazione, a un soggetto attivo, che è in rapporto dinamico con l'organizzazione. Il confine tra mondo sanitario e mondo dei "pazienti" non è managerialmente più sostenibile. Il manager sanitario di oggi deve far integrare nei percorsi diagnostici terapeutici assistenziali (PDTA) gli interventi di umanizzazione delle cure che presentano evidenze scientifiche. Per esempio, la music medicine è una delle strategie guida per il cambio del paradigma, per togliere il confine e per far sentire l'utente un soggetto che partecipa attivamente al suo percorso sanitario, in cui non esiste solo il suo corpo, ma in cui viene considerata e potenziata ogni sua risorsa, biologica, psicologica, culturale e sociale.

\section{Disclosures}

Financial support: The authors declare no financial support for this manuscript.

Conflict of interest: The authors declare no conflict of interest.

\section{Bibliografia}

1. Von Bertalanffy L. General system theory. George Braziller. New York. 1972.

2. Laszlo E. The systems view of the world. George Braziller. New York. 1968.

3. Decreto Legislativo 30 dicembre 1992 n. 502.

4. Decreto del Ministero della Sanità 15 ottobre 1996.

5. Decreto del Presidente del Consiglio dei Ministri 19 maggio 1995.

6. Costituzione Repubblicana Italiana.

7. Legge 7 agosto 1990 n. 241.

8. Patto per la Salute 2014-2016.

9. Atto di Indirizzo del Ministero della Salute 2017. 